\title{
Optimization of a HOT LWIR HgCdTe Photodiode for Fast Response and High Detectivity in Zero-Bias Operation Mode
}

\author{
M. KOPYTKO, ${ }^{1,3}$ A. KEBŁOWSKI ${ }^{2}$ P. MADEJCZYK, ${ }^{1}$ P. MARTYNIUK, ${ }^{1}$ \\ J. PIOTROWSKI ${ }^{2}$ W. GAWRON,${ }^{2}$ K. GRODECKI, ${ }^{1}$ K. JÓŹWIKOWSKI, ${ }^{1}$ \\ and J. RUTKOWSKI ${ }^{1}$ \\ 1.-Institute of Applied Physics, Military University of Technology, 2 Kaliskiego St., \\ 00-908 Warsaw, Poland. 2._Vigo System S.A., 129/133 Poznańska St., 05-850 Ożarów Mazowiecki, \\ Poland. 3.—e-mail: malgorzata.kopytko@wat.edu.pl
}

Fast response is an important property of infrared detectors for many applications. Currently, high-temperature long-wavelength infrared $\mathrm{HgCdTe}$ heterostructure photodiodes exhibit subnanosecond time constants while operating under reverse bias. However, nonequilibrium devices exhibit excessive low-frequency $1 / f$ noise that extends up to $\mathrm{MHz}$ range, representing a severe obstacle to their widespread application. Present efforts are focused on zero-bias operation of photodiodes. Unfortunately, the time constant of unbiased photodiodes is still at the level of several nanoseconds. We present herein a theoretical investigation of device design for improved response time and detectivity of long-wavelength infrared $\mathrm{HgCdTe}$ photodiodes operating at $230 \mathrm{~K}$ in zero-bias mode. The calculation results show that highly doped $p$-type $\mathrm{HgCdTe}$ is the absorber material of choice for fast photodiodes due to its high electron diffusion coefficient. The detectivity of such a device can also be optimized by using absorber doping of $N_{\mathrm{A}}=1 \times 10^{17} \mathrm{~cm}^{-3}$. Reduction of the thickness is yet another approach to improve the device response. Time constant below $1 \mathrm{~ns}$ is achieved for an unbiased photodiode with absorber thickness below $4 \mu \mathrm{m}$. A tradeoff between the contradictory requirements of achieving high detectivity and fast response time is expected in an optically immersed photodiode with very small active area.

Key words: HgCdTe photodiodes, uncooled photodetectors, photodiode response, time constant

\section{INTRODUCTION}

Infrared (IR) detectors can be used for a variety of applications in military, scientific, security, medical, industrial, and automotive areas. Common applications using IR technology include rail safety, gas leak detection, flame detection, petroleum exploration, heat distribution monitoring, medical diagnostic imaging, space operations, free-space optical communication, night vision, and spectroscopy. ${ }^{1-6}$ This wide range of applications implies the need for devices with different detection parameters. This, in turn, determines the type of IR photodetectors used,

(Received December 1, 2016; accepted April 28, 2017; published online May 25, 2017) which in general can be divided into two classes: thermal and photon (or quantum).

Thermal detectors operate by absorption of incoming radiation, which causes a change in the temperature of the detector, and by the sensitivity of some measurable parameter, for example, resistance, to that temperature. Common types of thermal detectors are bolometers, thermocouples, and pyroelectric detectors. Thermal detectors are typically sensitive within a wide range of incident wavelengths, and most of them are operated at room temperature. Due to their simple design, thermal detectors are characterized by not very high detectivity and relatively long time constant. For example, germanium bolometers are used at 
wavelengths between $5 \mu \mathrm{m}$ and $10 \mu \mathrm{m}$, and their thermal time constant is on the order of $10 \mathrm{~ms}^{7}$

Photon detectors depend on direct interaction of incoming light with detector materials, resulting, for example, in electron-hole pair creation in a semiconductor. Photogenerated carriers can be measured by directly measuring the charge collected during an integration period, by measuring the photocurrent, by a change in resistance (photoconductive), or by the voltage generated across a junction (photovoltaic). Photon detectors are produced using complex semiconductor materials and are characterized by extremely high performance. For the last 50 years, mercury cadmium telluride $\left(\mathrm{Hg}_{1-x} \mathrm{Cd}_{x} \mathrm{Te}\right)$ has been the main material for midwavelength infrared (MWIR, $3 \mu \mathrm{m}$ to $5 \mu \mathrm{m}$ ) as well as long-wavelength (LWIR, $8 \mu \mathrm{m}$ to $12 \mu \mathrm{m}$ ) infrared detectors. However, a problem facing photon detectors working in the IR region of the electromagnetic spectrum is that the energy of the absorbed photons of incident radiation is comparable to the thermal energy of the atoms in the detector itself. The statistical nature of thermal generation of charge carriers is a source of noise. There are many ways to overcome this problem, but cooling IR photon detectors to very low temperatures (often to liquidnitrogen temperature) is the most commonly used method.

Development of advanced epitaxial techniques such as molecular beam epitaxy (MBE) ${ }^{8}$ and metalorganic chemical vapor deposition (MOCVD) ${ }^{9}$ has enabled construction of complex HgCdTe heterostructure-based IR detectors for high-operating-temperature (HOT) conditions. ${ }^{4}$ The importance of research in the field of HOT detectors results from the possibility of cost reduction in production of infrared devices and their improved operability, thus extending their applications in industry, science, medicine, ecology, and especially portable applications, where cryogenic cooling is impossible or strongly limited.

Various ways to reduce thermal generation have been proposed ${ }^{10-12}$ for HOT conditions. Significant improvements have been achieved by reducing the absorber volume using optical immersion, ${ }^{4}$ doubleor multiple-pass IR radiation, ${ }^{4}$ suppression of Auger thermal generation in nonequilibrium photoconductors, ${ }^{13,14}$ photodiodes ${ }^{15-18}$ magnetoconcentration effect detectors, ${ }^{19,20}$ and recent barrier detectors. $^{21-24}$

Nonequilibrium devices require significant bias current and exhibit excessive low-frequency noise that extends up to the $\mathrm{MHz}$ range. This noise, which reduces detectivity, as well as high current requirements represent severe obstacles to their widespread application.

Our present efforts are focused on zero-bias operation of photodiodes. The resistance at zero bias determines the thermal noise level and ultimate detectivity that can be obtained when noise from other sources is negligible.
Currently, LWIR photodiodes exhibit subnanosecond time constants while operating under reverse bias. ${ }^{25,26}$ However, the time constant of unbiased photodiodes is still at the level of several nanoseconds. Achieving unbiased devices with fast response time requires a different detector design. We present herein a theoretical investigation on the influence of the active region thickness and its arsenic doping level on the time constant and current responsivity of LWIR HgCdTe photodiodes operating at near-room temperature in zero-bias mode.

\section{DEVICE DESIGN AND FABRICATION}

The investigated device is a photodiode based on $\mathrm{HgCdTe}$ multilayer heterostructures. Figure 1 shows schematic cross-sections of the analyzed mesa-structure photodiode. Typically, the modified $N^{+} p P^{+} \mathrm{HgCdTe}$ photodiode consists of:

- Infrared absorber with bandgap, composition, and thickness optimized for the best compromise between the requirements for efficient use of IR radiation and low thermal generation.

- Wide-bandgap and heavily doped $P^{+}$and $N^{+}$ contacts to minimize thermal generation and parasitic impedance.

- $n^{+}$-cap layer to provide low-resistance tunnel contact connecting $P^{+}$and top mesa metallization.

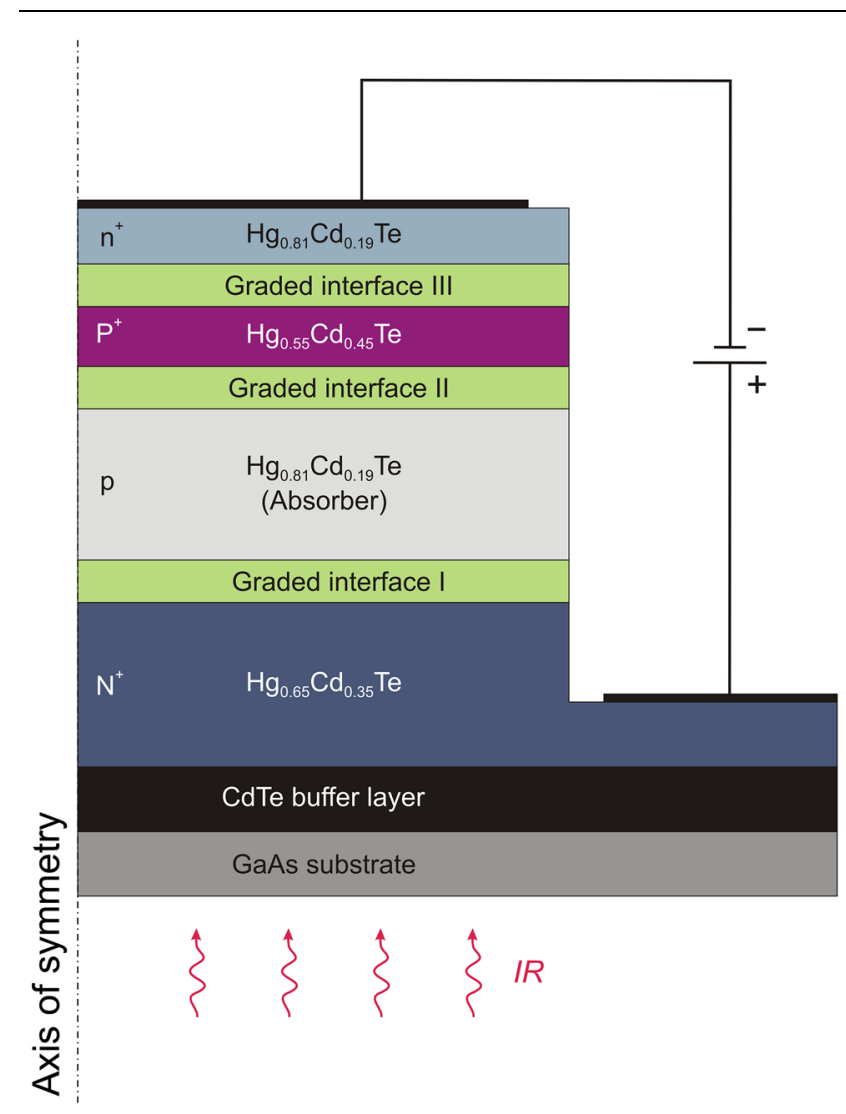

Fig. 1. Schematic structure of modified LWIR $N^{+} p P^{+} \mathrm{HgCdTe}$ heterojunction photodiode. 
- Graded-bandgap interfaces to suppress tunneling and thermally generated currents.

- Metal-semiconductor contacts.

The device is backside-illuminated through the $\mathrm{N}^{+}$layer, which plays the role of an infraredtransmitting window for photons with energy below the bandgap. Since the diffusion length in the $N^{+}$ layer is short compared with its thickness, charge carriers generated in the layer do not produce photocurrent. The bandgap of the layer determines the cut-on wavelength of the device.

Devices were fabricated in a joint laboratory run by VIGO System S.A. and Military University of Technology (MUT), Warsaw, Poland. HgCdTe epilayers were grown on 2-inch, epi-ready, semiinsulating (100) GaAs substrates using the MOCVD technique. Typically, a 3-4- $\mu$ m-thick CdTe layer was used as a buffer layer to reduce stress caused by crystal lattice misfit between the GaAs substrate and $\mathrm{HgCdTe}$ epitaxial layer structure. This buffer also played the role of a Ga diffusion barrier.

Growth was typically carried out at temperature of about $350^{\circ} \mathrm{C}$ with mercury zone at $210^{\circ} \mathrm{C}$ using the interdiffused multilayer process (IMP) in a horizontal, near-atmospheric-pressure MOCVD AIX 200 reactor. The system was designed to operate in laminar flow regime with process pressure from 50 mbar to 1000 mbar using a butterfly valve for pressure control. Reactor pressure of 500 mbar was used for all successful growth runs. Hydrogen was used as carrier gas. Dimethylcadmium (DMCd) and diisopropyltelluride (DIPTe) were used as precursors. Ethyl iodine (EI) was used as donor, and trisdimethylaminoarsine (TDMAAs) as an acceptor of dopant sources. DMCd and EI were delivered through one channel, while DIPTe and TDMAAs were delivered through another channel over the quartz container where elementary mercury was held. Detailed description of the MOCVD growth procedure is presented in Ref. 27.

Practical MOCVD-grown $\mathrm{HgCdTe}$ single-pixel devices were fabricated with mesa-geometry structure using standard photolithography and wet chemical etching to form an electric contact to the bottom contact layer. Devices were monolithically integrated with GaAs microlenses as optical concentrators. Use of hyperhemispherical immersion lenses significantly improves the performance of HOT photodiodes. The main advantages include ${ }^{4}$ :

- Improved ratio of junction to series resistance: although the junction resistance is to a first approximation proportional to the area, the series resistance is less dependent on area.

- Reduced capacitance by a factor of $n^{4}$, where $n$ is the refractive index of the lens material, resulting in significantly increased cut-off frequency for $R C$-limited devices.
- Improved detectivity by a factor of $n^{2}$ due to the increased optical area of the detector.

\section{METHOD OF ANALYSIS}

We conducted two-dimensional (2D) modeling of the mesa geometry structure shown in Fig. 1. Our computer software is based on a solution of the set of transport equations comprising the continuity equations for electrons and holes, Poisson's equation, and thermal conductivity equation, given by ${ }^{28,29}$

$$
\begin{gathered}
\frac{\partial n}{\partial t}=\frac{1}{q} \nabla \overrightarrow{j_{n}}+G-R, \\
\frac{\partial p}{\partial t}=-\frac{1}{q} \nabla \overrightarrow{j_{p}}+G-R, \\
\nabla^{2} \Psi=-\frac{q}{\varepsilon \varepsilon_{0}}\left[N_{\mathrm{D}}^{+}-N_{\mathrm{A}}^{-}+p-n\right]-\frac{1}{\varepsilon} \nabla \Psi \nabla \varepsilon, \\
C_{V} \frac{\partial T}{\partial t}-H=-\nabla(\chi \cdot \nabla T),
\end{gathered}
$$

where $\Psi$ is the electrostatic potential, $j$ is the current density, $q$ is the elementary charge, $C_{V}$ is the specific heat, $\chi$ is the thermal conductivity coefficient, $T$ is temperature, $G$ is the generation rate, $R$ is the recombination rate, and $H$ is the heat generation term. The indices $n$ and $p$ denote electron and hole, respectively. In the last term, the Joule heat is introduced as heat generation to include thermoelectric phenomena and the heat balance. The difference $G-R$ is the net generation of electron-hole pairs and depends on all phenomena including the influence of thermal as well as tunneling mechanisms.

To determine the response time of the photodiode, the Fourier expansion method was applied. The method consists of describing the carrier transport equations in complex space and computing all the photoelectric parameters of the structure, including the currents flowing in steady-state condition at definite bias voltage. Then, after activating the structure by a small harmonic optical pulse with frequency $\omega$, the complex amplitude of the photocurrent is calculated, and the real part of the obtained photocurrent amplitude is normalized by dividing by the value of the photocurrent for frequency $\omega=0$ to describe the frequency response of the photodiode. In the case of the simplest detector, the time constant $\tau$, defined by the cut-off frequency $f_{T}$ at the $-3-\mathrm{dB}$ line, can be calculated as

$$
\tau=\frac{1}{2 \pi f_{T}}
$$

There are more complex cases where the detector time response is limited by different mechanisms, 
such as different carrier recombination mechanisms, space charge, and diffusion capacitance..$^{30}$

In the calculations, the wavelength of the incident light was assumed to be $\lambda_{\text {inc }}=8.5 \mu \mathrm{m}$ and the power density to be $P_{\text {inc }}=0.1 \mathrm{~W} / \mathrm{cm}^{2}$. Detailed description of the method is presented in our previous paper. ${ }^{31}$ Numerical analysis of the frequency response of the photodiode used an approach slightly different from that applied in the time domain. ${ }^{32}$

At the boundaries of this domain, appropriate conditions must be specified. In the vicinity of the semiconductor surface on which the metal electrical contacts were deposited, the boundary conditions for the electron and hole currents are defined by

$$
\begin{gathered}
j_{n}=q S\left(n-n_{0}\right), \\
j_{p}=-q S\left(p-p_{0}\right),
\end{gathered}
$$

where $S$ is the surface recombination velocity parameter. We considered account ohmic contacts with $S=10^{6} \mathrm{~cm} / \mathrm{s}$, similar to in Refs. 33 and 34, and nonohmic contacts with $S=10^{2} \mathrm{~cm} / \mathrm{s}$.

\section{DEVICE OPTIMIZATION}

The time constant determines a detector's ability to distinguish successive incoming signals at short intervals. Depending on the type and application of the detector, there are various definitions of the time constant. Physically, the time constant represents the time required for the detector's response decrease to reach 1/e of its initial value.

Fast response is an important parameter for quantum IR detectors for use in many applications. It can be achieved in two different ways:

- Fast decay of photogenerated carriers due to rapid recombination in the absorber region.

- Fast transport of photogenerated carriers to collection contacts.

Since the recombination mechanism is a statistical process and leads to noise and poor signal-to-noise performance, the first approach is not optimal for HOT detectors. However, this method is used in many fast-response devices, including photoconductors. Therefore, only the second solution finds practical application for photodiodes. The transport of photogenerated carriers to contacts in complex heterostructure devices, as presented in Fig. 1, is determined by:

- The drift transit time $\left(\tau_{\mathrm{dr}}\right)$ across the spacecharge regions (depletion area).

- The diffusion time $\left(\tau_{\text {diff }}\right)$ from the place of generation to the space-charge regions.

The drift transit time across the space-charge regions can be expressed as

$$
\tau_{\mathrm{dr}}=\frac{w}{\mu_{\mathrm{a}} E}=\frac{w^{2}}{\mu_{\mathrm{a}} V_{\mathrm{b}}},
$$

where $w$ is the width of the space-charge region, $\mu_{\mathrm{a}}$ is the ambipolar carrier mobility, $E$ is the electric field, and $V_{\mathrm{b}}$ is the bias voltage. The ambipolar carrier mobility can be expressed as

$$
\mu_{\mathrm{a}}=\frac{\mu_{\mathrm{h}} \mu_{\mathrm{e}} n-\mu_{\mathrm{e}} \mu_{\mathrm{h}} p}{\mu_{\mathrm{e}} n+\mu_{\mathrm{h}} p}
$$

where $\mu_{\mathrm{e}}$ and $\mu_{\mathrm{h}}$ are the electron and hole mobility, and $n$ and $p$ are the electron and hole concentration, respectively. Due to the high mobility of electrons and low mobility of holes $\left(\mu_{\mathrm{e}} / \mu_{\mathrm{h}}=100\right)$, fast-response devices should be designed using an absorbing layer in which the minority carriers are electrons. A $p$-type absorber is advantageous for obtaining time constant on the order of $100 \mathrm{ps}$.

If generation takes place at a distance from the space-charge region of less than the ambipolar diffusion length $\left(l \ll L_{\mathrm{D}}\right)$, the diffusion-limited transit time can be expressed as

$$
\tau_{\text {diff }}=\frac{l^{2}}{2.4 D_{\mathrm{a}}},
$$

where $D_{\mathrm{a}}$ is the ambipolar diffusion coefficient:

$$
D_{\mathrm{a}}=\frac{D_{\mathrm{h}} \mu_{\mathrm{e}} n+D_{\mathrm{e}} \mu_{\mathrm{h}} p}{\mu_{\mathrm{e}} n+\mu_{\mathrm{h}} p},
$$

where $D_{\mathrm{e}}$ and $D_{\mathrm{h}}$ are the diffusion coefficients for electrons and holes.

According to Eq. 10, high ambipolar diffusion coefficient shortens the time needed for carriers to reach the junctions. The calculated dependence of the ambipolar diffusion coefficient divided by the electron diffusion coefficient on the $p$-type doping is shown in Fig. 2. The electron-to-hole mobility ratio is assumed to be $\mu_{\mathrm{e}} / \mu_{\mathrm{h}}=100$. For $p$-type material, in which $p \gg n_{\mathrm{i}}$, the ambipolar diffusion coefficient equals the electron diffusion coefficient. If $p=n_{\mathrm{i}}$, the ambipolar diffusion coefficient is only $10 \%$ of the electron diffusion coefficient, while for doping level of $p=3 n_{\mathrm{i}}$, the ambipolar diffusion coefficient is about half the electron diffusion coefficient. Thus, use of a highly doped $p$-type absorber significantly improves the diffusion transit time.

Another important factor influencing the response time of IR detectors is the $R C$ time constant $\left(\tau_{R C}\right)$, which is equal to the product of the serial circuit resistance $R$ and capacitance $C$ :

$$
\tau_{R C}=R C .
$$

Beyond the resistance of the space-charge regions, there are other resistances which to a first approximation can be considered as linear series resistances:

- Resistance of metallization.

- Resistance of metal-semiconductor contact. 


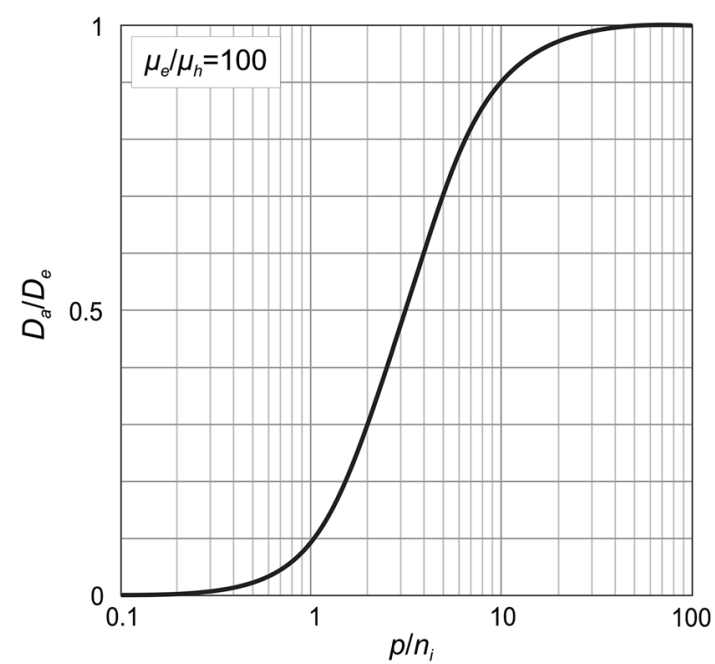

Fig. 2. Calculated dependence of ambipolar diffusion coefficient over electron diffusion coefficient on $p$-type doping, assuming electron-to-hole mobility ratio of $\mu_{\mathrm{e}} / \mu_{\mathrm{h}}=100$. The relation is valid for near-room temperature and low molar composition of $\mathrm{Hg}_{1-x} \mathrm{Cd}_{x} \mathrm{Te}$.

- Resistance of active layer and contacts.

- Resistance of electrical connections.

There are two types of photodiode capacitance: the space-charge region (or junction) capacitance, and the diffusion capacitance related to the charge of the minority carriers in the neutral diode region. ${ }^{30}$ Since the minority carrier concentration decreases rapidly with reverse bias, the diffusion capacitance can be greatly reduced in reversebiased devices. Unfortunately, the diffusion capacitance is very large in an unbiased narrow-gap absorber, where the minority carrier concentration is high at elevated operation temperatures, greatly affecting the response time of the device. ${ }^{32}$ To some degree, the diffusion-related capacitance time constant can be minimized by reduction of the series resistance of the device and the input resistance of the preamplifiers.

Figure 3 shows experimental data for the time constant as a function of reverse bias voltage, measured for LWIR HgCdTe photodiodes characterized by different series resistances. Increasing the reverse bias initially increases the response time to a maximum value above $10 \mathrm{~ns}$. However, further increase of the bias reduces the time constant by about two orders of magnitude. The time constants become quite short for biases higher than the threshold voltage. Devices exhibit subnanosecond time constants when operating under high reverse bias. ${ }^{25,26}$ However, the time constant of unbiased photodiodes is still at the level of several nanoseconds. At zero bias, electron-hole pairs are generated in the diffusion layer, which is practically free of electric field. The detector's response time is mainly limited by the diffusion time of carriers from

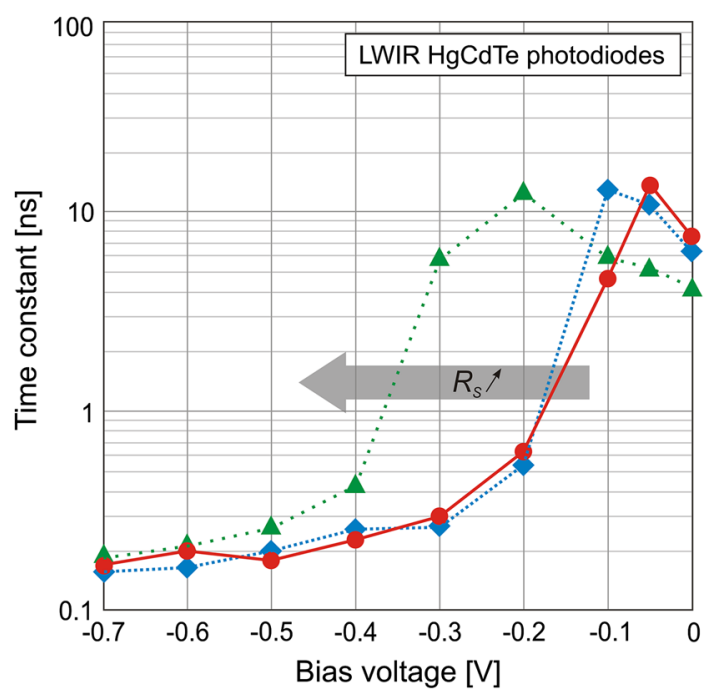

Fig. 3. Time constant of photodiodes with different series resistances measured as function of reverse bias voltage. Series resistance increases in the direction of the arrow.

the place of generation to the space-charge regions, as well as the $R C$ time constant.

The main objective of this study is to achieve a tradeoff between the contradictory requirements of high detectivity and fast response time under zerobias conditions. Structural parameters such as the contact, barrier, and absorber doping, absorber and barrier composition, and absorber thickness were changed in the calculations (Table I) to determine the optimal device architecture.

The assumed composition and doping concentration profiles permit us to calculate the energy bandgap. Figure 4 shows the simulated energy band diagrams and distribution of the electric field for an unbiased and reverse-biased $N^{+} p P^{+} \mathrm{HgCdTe}$ heterojunction photodiode operated at $230 \mathrm{~K}$. CB and VB denote conduction and valence bands, respectively. The built-in electric field is mainly associated with the junction interfaces, and the maximum electric field appears at the $N^{+}$-absorber heterojunction. Under reverse bias, the electric field intensity is almost two times higher in a device with heavily doped absorber, whereas for low absorber doping, the electric field extends deeper into the active layer.

Figure 5 shows the frequency response calculated for the photodiode with a $3-\mu \mathrm{m}$-thick absorber for different applied reverse biases and doping levels. The cut-off frequency $f_{T}$ is depicted by the $-3-\mathrm{dB}$ line. For a photodiode with highly doped absorption region, $f_{T}$ is two times higher than for a photodiode with lightly doped absorber region. In both cases, reverse biasing shifts the cut-off frequency towards higher values.

The time constant and peak current responsivity as functions of the reverse bias voltage are shown in Fig. 6. The calculations were carried out for ohmic and nonohmic metal-semiconductor contacts. Use of nonohmic contacts greatly deteriorated both the 
Table I. Structural parameters for modeling of modified LWIR $N^{+} p P^{+}$HgCdTe heterojunction photodiode

\begin{tabular}{|c|c|c|c|}
\hline & Thickness, $d(\mu \mathrm{m})$ & Composition, $x$ & Dopant concentrations, $N_{\mathrm{D}}, N_{\mathrm{A}}\left(\mathrm{cm}^{-3}\right)$ \\
\hline Contact layer $\left(n^{+}\right)$ & 1 & 0.19 & $1 \times 10^{18}$ \\
\hline Graded interface III & 0.2 & & \\
\hline Barrier layer $\left(P^{+}\right)$ & 0.9 & $0.2-0.45$ & $5 \times 10^{17}$ \\
\hline Graded interface II & 0.5 & & \\
\hline Absorber $(p)$ & $1-9$ & 0.19 & $1 \times 10^{15}-1 \times 10^{17}$ \\
\hline Graded interface I & 0.4 & & \\
\hline Contact layer $\left(N^{+}\right)$ & 8 & 0.35 & $2 \times 10^{17}$ \\
\hline
\end{tabular}
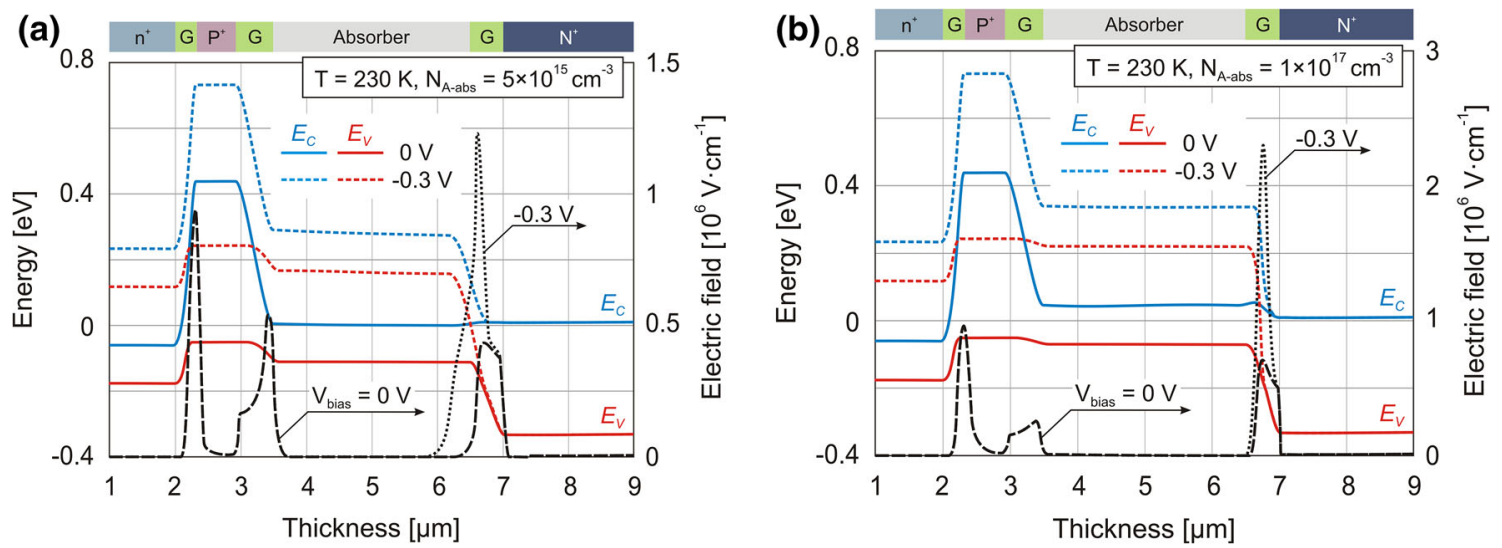

Fig. 4. Calculated energy band diagrams and spatial distribution of built-in electric field for unbiased (solid lines) and reverse-biased (dashed lines) photodiodes with absorber doping level of (a) $N_{\mathrm{A}}=5 \times 10^{15} \mathrm{~cm}^{-3}$ and (b) $N_{\mathrm{A}}=1 \times 10^{17} \mathrm{~cm}^{-3}$. Layer thickness measured along axis of symmetry from surface to substrate.
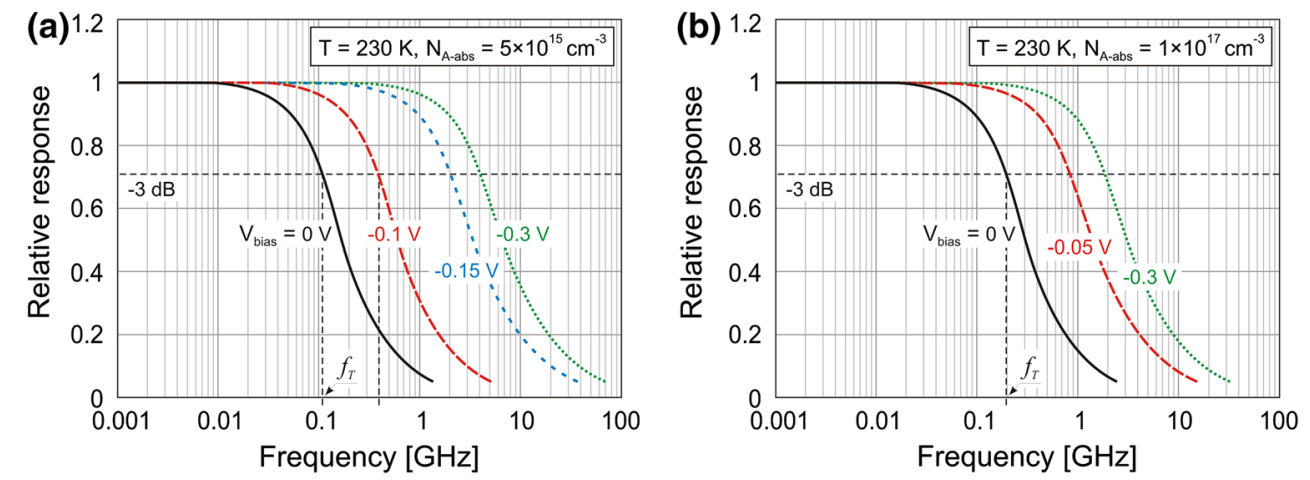

Fig. 5. Calculated frequency response for unbiased and reverse-biased photodiodes with absorber doping level of (a) $N_{\mathrm{A}}=5 \times 10^{15} \mathrm{~cm}^{-3}$ and (b) $N_{\mathrm{A}}=1 \times 10^{17} \mathrm{~cm}^{-3}$.

time response and current responsivity of the device as well as increasing the threshold voltage (Fig. 7) due to the voltage drop at contacts. Therefore, lowresistance, stable contacts to semiconductors are critical to the device performance.

Under equilibrium conditions at zero bias, lightly doped material $\left(N_{\mathrm{A}} \leq 3 \times 10^{16} \mathrm{~cm}^{-3}\right)$ is characterized by low ambipolar mobility and diffusion coefficient, resulting in slow carrier transport. Concurrently, the recombination time in the material is quite short, mostly being due to the Auger 1 process. Therefore, a significant portion of the carriers recombine before reaching the contacts. As a consequence, zero-biased devices also suffer from low responsivity.

The time constant of a photodiode with lightly doped absorber initially increases with low reverse bias, reaching its maximum value at bias close to that at which the maximum dark current occurs. With higher reverse voltage, the response time significantly decreases as the result of improved collection of photogenerated carriers due to extraction of minority electrons from the absorber, which results in increased ambipolar mobility. 

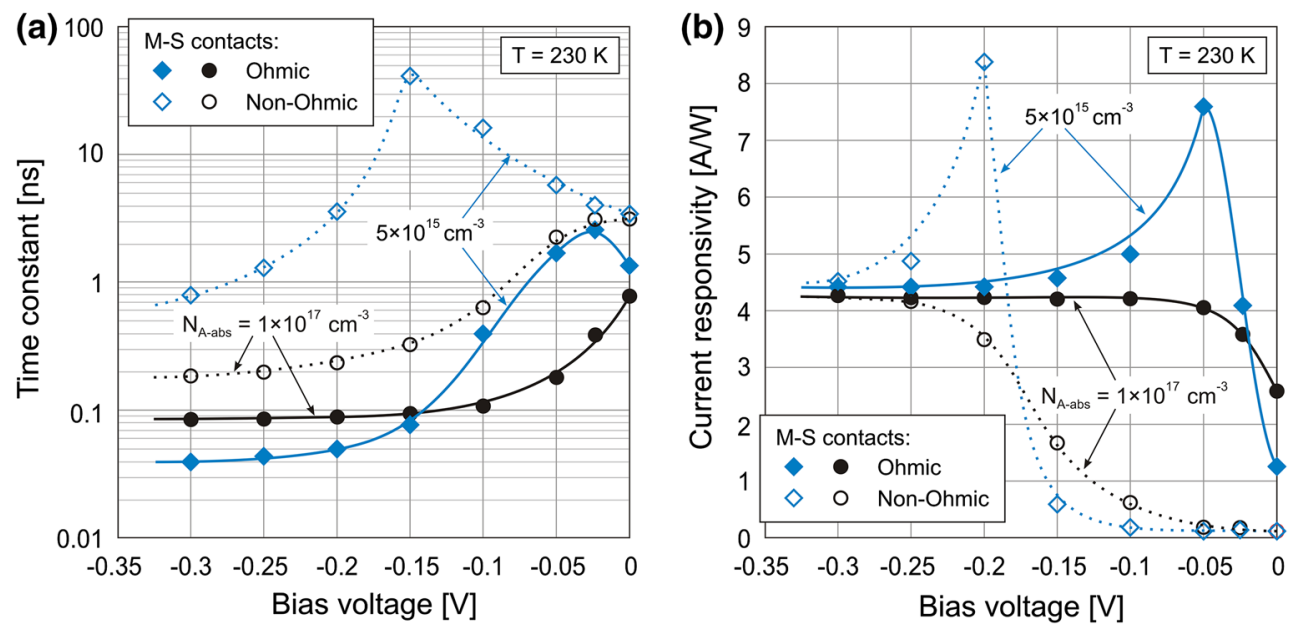

Fig. 6. Time constant (a) and current responsivity (b) as functions of reverse bias voltage, calculated for two types of metal-semiconductor contacts: ohmic (solid lines) and nonohmic (dotted lines).

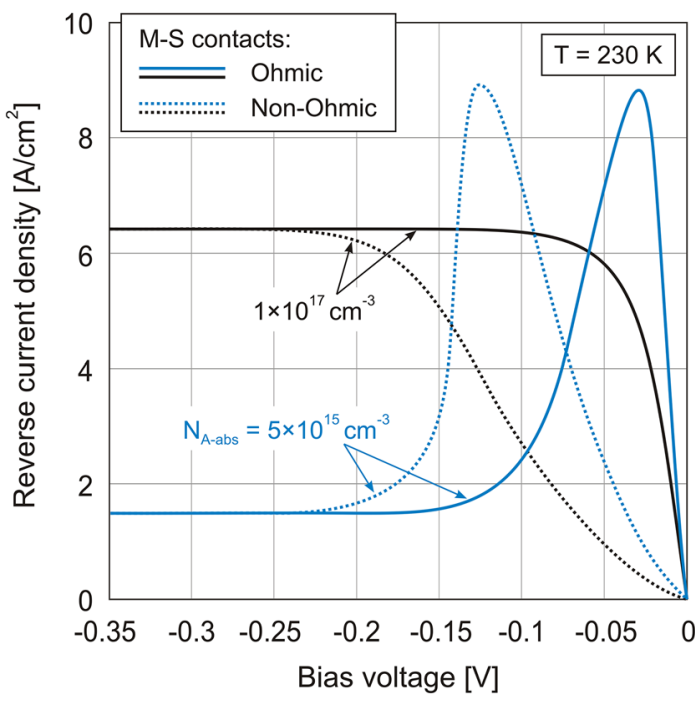

Fig. 7. Current-voltage characteristic calculated for two types of metal-semiconductor contacts: ohmic (solid lines) and nonohmic (dotted lines).

The photodiode also shows a maximum current responsivity value in the negative resistance region. The puzzling effect is the large photocurrent in the negative resistance bias range (Fig. 8). The measured quantum efficiency often exceeds $100 \%$. This can be explained by mixed conductivity effects, impact ionization, and lateral collection. ${ }^{35}$ Piotrowski's $^{36}$ explanation based on numerical simulation of photocurrent generation is the following:

- Collection of photogenerated charge carriers for small and intermediate (negative resistance range) voltage is weak, so illumination increases the concentration of charge carriers in the absorber.

- The increased concentration of charge carriers results in a further increase of the current concentration due to Auger generation.

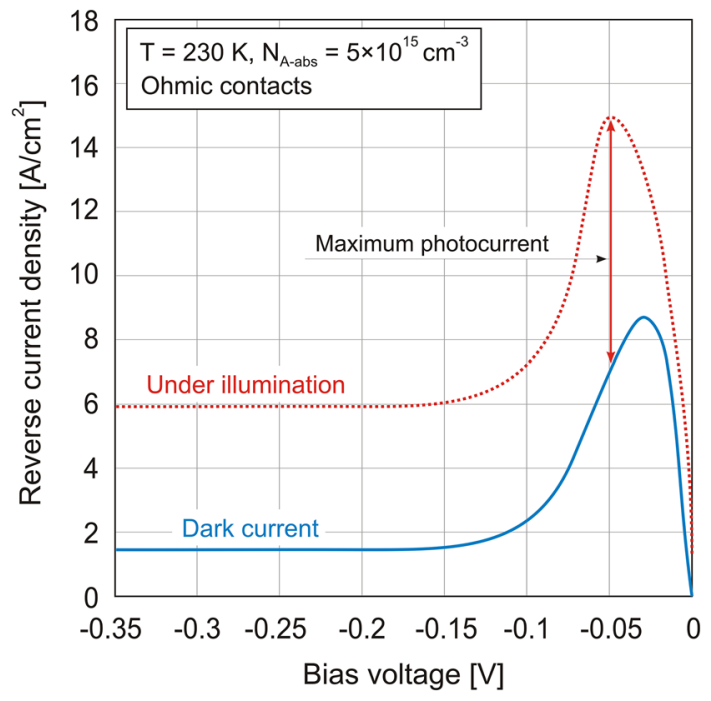

Fig. 8. Current-voltage characteristic of analyzed photodiodes calculated in dark and under illumination.

- In the negative resistance range of bias voltages, higher current causes an increased voltage drop across the series resistance, which reduces the voltage within the structure and results in a further current increase. The practical consequence is generation of a current larger than that corresponding to $100 \%$ quantum efficiency.

- At large voltages, transport becomes very efficient, rapidly collecting photogenerated carriers to contacts. This prevents the increased carrier concentration and related Auger generation, reduces the voltage drop across the series resistance, and results in internal quantum efficiency close to $100 \%$.

For a photodiode with highly doped absorption region, the current density increases with a reverse bias. With increase of the bias voltage, the carrier 
lifetime and current responsivity also increase, both saturating at a certain threshold voltage. The time constant decreases with reverse bias, as in the case of the device with lightly doped absorber region. However, heavily doped absorber provides slower response under reverse bias conditions. This is caused by the smaller depletion of the heavily doped absorber region.

The performance of zero-biased devices can be improved by proper design of the detection structure, doping of the absorber to level of $p \approx 3 n_{\mathrm{i}}$, ${ }^{4}$ use of an optimal absorber thickness, and reduction of parasitic capacitances and resistances.

The normalized detectivity can be determined from the current responsivity $\left(R_{\mathrm{i}}\right)$ and the noise current $\left(i_{\mathrm{n}}\right) \mathrm{as}^{22}$

$$
D^{*}=\frac{R_{\mathrm{i}}}{i_{\mathrm{n}}} \sqrt{A}
$$

where $A$ is the detector area. The resistance at zero bias determines the thermal noise level. The zerobias detectivity due to thermal Johnson-Nyquist noise can then be expressed as

$$
D^{*}=\frac{R_{\mathrm{i}}}{\sqrt{4 k_{\mathrm{B}} T / R_{0} A}}
$$

where $R_{0}$ is the resistance at zero bias, $T$ is temperature, and $k_{\mathrm{B}}$ is the Boltzmann constant. The normalized detectivity at zero bias voltage depends on the current responsivity and $R_{0} A$ product.

Figure 9 shows the time constant and peak detectivity as functions of the absorber doping of the $N^{+} p P^{+} \mathrm{HgCdTe}$ photodiode operated at zero bias. Calculations were carried out for a 3- $\mu \mathrm{m}$-thick absorber layer and assuming ideal ohmic contacts. For acceptor concentration in the absorber up to the intrinsic concentration $\left(2 \times 10^{16} \mathrm{~cm}^{-3}\right)$, the time constant of the photodiode assumes a constant value of about $1.5 \mathrm{~ns}$. As expected from Fig. 2, the time constant decreases below $1 \mathrm{~ns}$ for higher $p$-type doping. Moreover, higher doping in the absorber region also improves the detectivity of the photodiode. On increasing the doping in the absorber from $N_{\mathrm{A}}=1 \times 10^{15} \mathrm{~cm}^{-3}$ to $N_{\mathrm{A}}=1 \times 10^{17} \mathrm{~cm}^{-3}$, the detectivity increases by about an order of magnitude. Doping level of $p \approx 3 n_{\mathrm{i}}$ is optimal due to the minimized Auger thermal generation and increased quantum efficiency resulting from the longer diffusion length in the more heavily doped material. ${ }^{4}$

Figure 10 shows the time constant and peak detectivity as functions of the absorber thickness of the analyzed photodiode operated at zero bias. Calculations were carried out for two doping levels of the absorber. As expected, the time constant of the photodiode decreases with a reduction of the thickness of the absorption region. Time constants below $1 \mathrm{~ns}$ can be obtained in unbiased photodiodes with thin absorption region. However, too thin an absorber results in poor detectivity of the device due

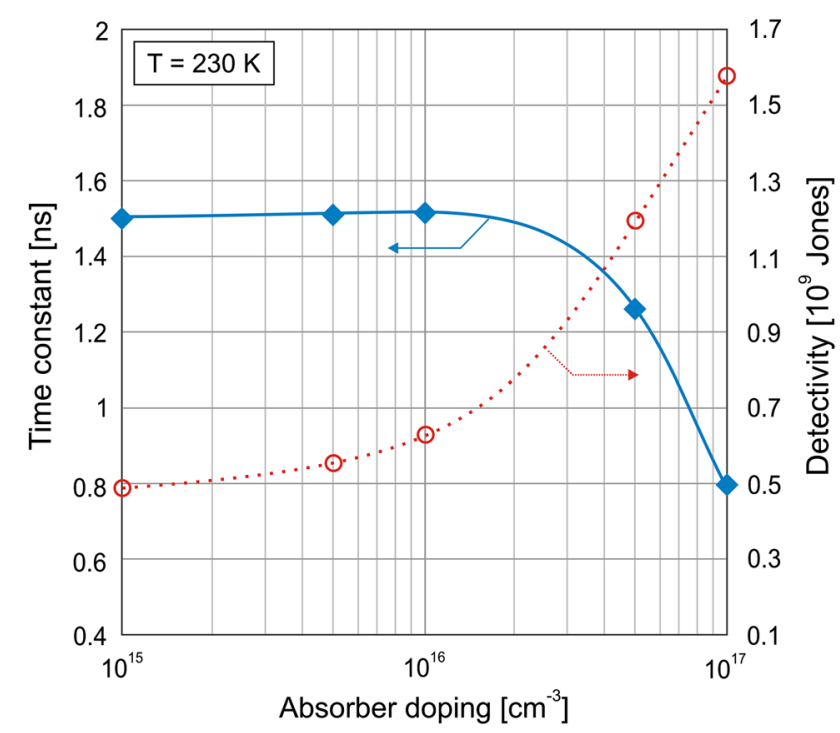

Fig. 9. Time constant and peak detectivity of analyzed photodiodes as functions of absorber doping in zero-bias operation.

to low quantum efficiency caused by only partial absorption of incoming photons. On the other hand, the absorption region cannot be too thick. In the LWIR range, the minority carrier lifetime is quite short, mostly being due to the Auger 1 process in the near-intrinsic material $\left(N_{\mathrm{A}} \leq 3 \times 10^{16} \mathrm{~cm}^{-3}\right)$. A significant portion of the carriers recombine before reaching the contacts, and only a part of the charge carriers photogenerated at distance shorter than the diffusion length from the junction can be collected effectively. The maximum quantum efficiency is achieved by devices with $3-\mu \mathrm{m}$-thick absorber layer doped at low level or a $4-\mu \mathrm{m}$-thick absorber doped at higher level; however, the maximum detectivity corresponds to $2 \mu \mathrm{m}$ for both doping levels.

Optimization of the graded interfaces is yet another issue. Proper design of graded interfaces between the main layers prevents formation of barriers in the conduction and valence bands. Each discontinuity of the bands may have an adverse effect on the device properties, giving rise to spikes in the charge carrier concentrations, large thermal generation rate, and excessive electric field. The practical consequence of energy barriers is large time constant and low detectivity due to blockage of collection of photogenerated carriers. These problems can be prevented by proper grading of absorber interfaces. Although significant grading has been observed in $\mathrm{HgCdTe}$ structures grown at the MOCVD growth temperature $\left(350^{\circ} \mathrm{C}\right)$, the interfaces frequently require additionally smoothing, which can be achieved by programmed growth. ${ }^{3}, 38$

The thickness of the $\mathrm{N}^{+}$- and $P^{+}$-layers should be optimized for low resistance. The top contact $n^{+}$ layer should be thin, in contrast to the bottom $N^{+}$, 

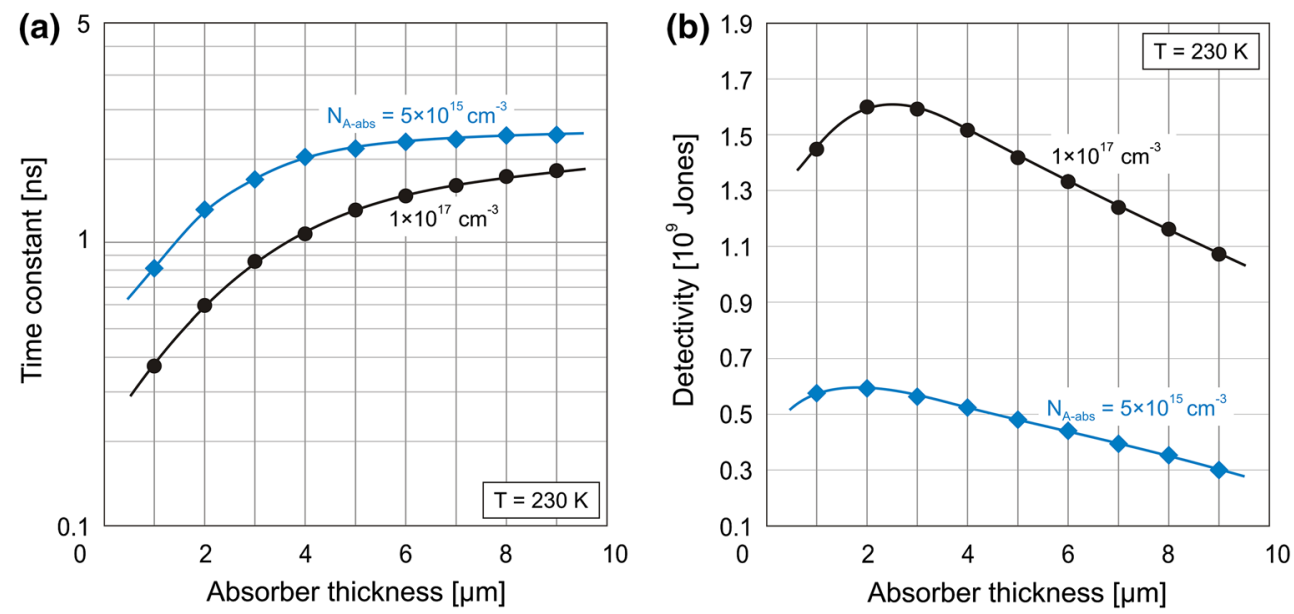

Fig. 10. Time constant (a) and peak detectivity (b) as functions of absorber thickness in zero-bias operation.
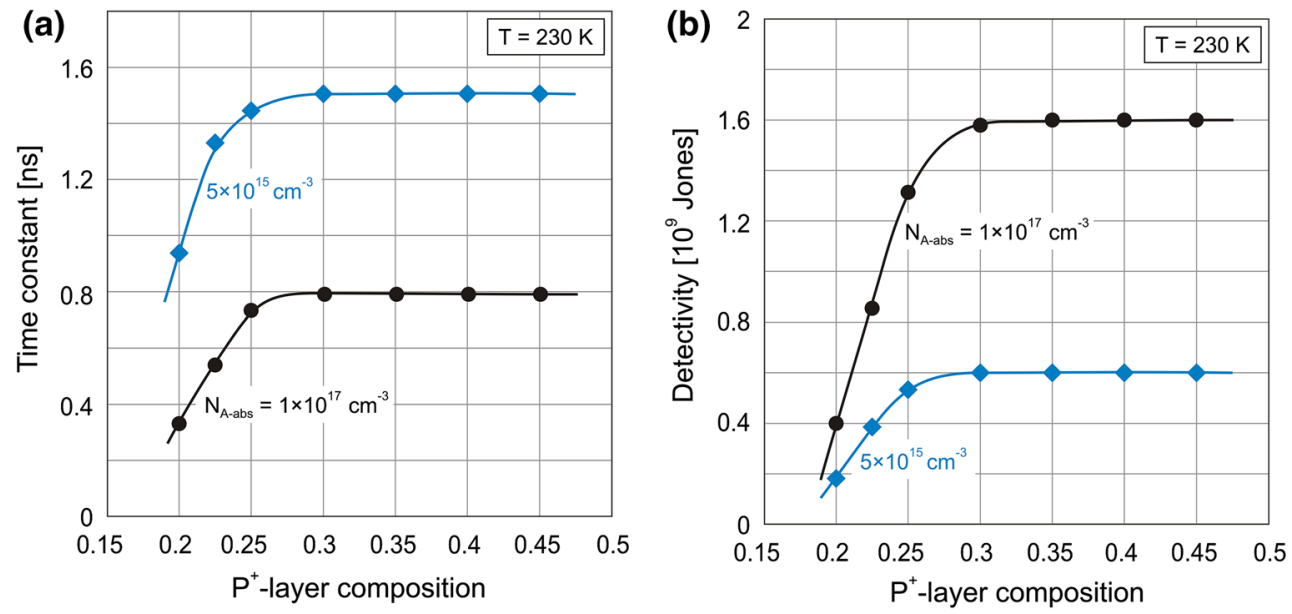

Fig. 11. Time constant (a) and peak detectivity (b) as functions of Cd molar composition of $P^{+}$layer.

which should be a few micrometers thick. Contact layers should be highly doped for low series resistance, low minority carrier concentration, and most importantly, easy formation of low-resistance contact to metallization. For HgCdTe layers, $n$-type doping with iodine is effective up to $10^{18} \mathrm{~cm}^{-3}$, while $p$-type doping with arsenic is limited to low $10^{17} \mathrm{~cm}^{-3}$. However, too heavy doping deteriorates the crystalline properties of $\mathrm{HgCdTe}$ epilayers.

Figure 11 shows the time constant and peak detectivity as functions of the $\mathrm{Cd}$ molar composition of the $P^{+}$layer from the base value of $x=0.45$ to $x=0.2$. Neither the time constant nor detectivity change on reducing the barrier layer composition to $x=0.3$. For lower molar composition of the $P^{+}$layer, the time constant significantly decreases, reaching its minimum for the homojunction device. However, the detectivity also decreases due to the reduced current responsivity and increased dark current in the homojunction device.
Figure 12 shows the calculated time constant and peak detectivity as functions of the mesa diameter, for zero bias. The time constant increases with increasing mesa diameter, mostly due to the fact that the electrical capacitance increases in proportion to the device area while the series resistance depends only weakly on device area. Increasing the mesa diameter also deteriorates the detectivity. This is caused by the higher ratio of series resistance to parallel device resistances. Thus, small pixel size makes achieving fast response and better performance easier.

\section{CONCLUSIONS}

The frequency response of unbiased $\mathrm{HgCdTe}$ heterostructure photodiodes is limited by two processes: transport through the absorber region and the $R C$ time constant. Highly doped $p$-type HgCdTe is the absorber material of choice for fast 

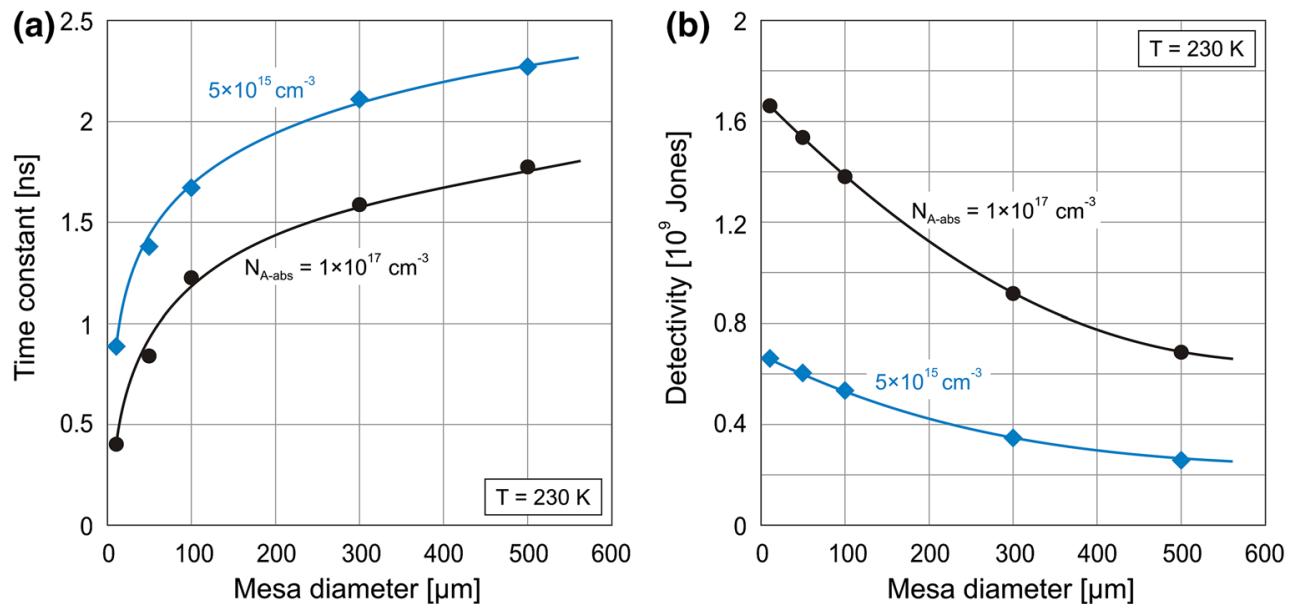

Fig. 12. Time constant (a) and peak detectivity (b) as functions of detector mesa diameter in zero-bias operation, calculated for two absorber doping levels.

photodiodes due to its large ambipolar diffusion coefficient for charge carriers. Devices with absorber doping of $N_{\mathrm{A}}=1 \times 10^{17} \mathrm{~cm}^{-3}$ have optimal detectivity due to reduced Auger thermal generation and longer ambipolar diffusion length. Further time constant reduction can be achieved by using a thin absorber. Time constant below $1 \mathrm{~ns}$ can be achieved in the unbiased photodiode with absorber doping of $N_{A}=1 \times 10^{17} \mathrm{~cm}^{-3}$ and absorber thickness below $4 \mu \mathrm{m}$. To obtain a time constant of $1 \mathrm{~ns}$ for absorber doping of $N_{\mathrm{A}}=5 \times 10^{15} \mathrm{~cm}^{-3}$, its thickness should be less than $1.5 \mu \mathrm{m}$, although the peak detectivity will decrease threefold.

The high-frequency performance of practical devices is often degraded by the equivalent $R C$ time constant due to built-in or external series resistance. This effect is especially pronounced in largearea devices. Low-resistance, stable ohmic contacts to semiconductors are critical to device performance. Reduction of the detector mesa diameter from $500 \mu \mathrm{m}$ to $50 \mu \mathrm{m}$ makes the time constant almost two times shorter.

\section{ACKNOWLEDGEMENTS}

We acknowledge support from The National Centre for Research and Development (Poland) through Grant No. TANGO1/2665576/NCBR/2015.

\section{OPEN ACCESS}

This article is distributed under the terms of the Creative Commons Attribution 4.0 International License (http://creativecommons.org/licenses/by/4.0/), which permits unrestricted use, distribution, and reproduction in any medium, provided you give appropriate credit to the original author(s) and the source, provide a link to the Creative Commons license, and indicate if changes were made.

\section{REFERENCES}

1. B.F. Jones, IEEE Trans. Med. Imag. 17, 1019 (1998).
2. D.M. Hepburn and I.J. Kemp, Eng. Sci. Edu. J. 7, 107 (1998).

3. P. Norton, Encyclopedia of Optical Engineering, ed. R. Driggers (New York: Marcel Dekker, 2003), pp. 320-348.

4. J. Piotrowski and A. Rogalski, High-Operating-Temperature Infrared Photodetectors (Bellingham: SPIE, 2007).

5. J. Wojtas, Z. Bielecki, T. Stacewicz, J. Mikołajczyk, and M. Nowakowski, Opto-Electron. Rev. 20, 26 (2012).

6. J. Hodgkinson and R.P. Tatam, Meas. Sci. Technol. 24, $012004(2013)$

7. H.C. Wang, X. Yi, G. Huang, J. Xiao, X.W. Li, and S.H. Chen, Infrared Phys. Technol. 45, 53 (2004).

8. T.J. de Lyon, R.D. Rajavel, J.A. Roth, and J.E. Jensen, Handbook of Infrared Detection and Technologies, ed. M. Henini and M. Razeghi (Oxford: Elsevier, 2002), pp. 309-352.

9. P. Mitra, F.C. Case, and M.B. Reine, J. Electron. Mater. 27, 510 (1998).

10. J. Piotrowski, W. Galus, and M. Grudzień, Infrared Phys. 31, 1 (1991).

11. J. Piotrowski, Infrared Photon Detectors, ed. A. Rogalski (Bellingham: SPIE Optical Engineering Press, 1995), pp. 391-493.

12. J. Piotrowski and A. Rogalski, Narrow-Gap II-VI Compounds and Electromagnetic Applications, ed. P. Capper (London: Chapman \& Hall, 1997), pp. 506-525.

13. M. White, Infrared Phys. 25, 729 (1985).

14. T. Ashley and C.T. Elliott, Electron. Lett. 21, 451 (1985).

15. C.T. Elliott and N.T. Gordon, Handbook on Semiconductors, vol. 4, ed. C. Hilsum (Amsterdam: North-Holland, 1993), pp. 841-936.

16. C.T. Elliott, Semicond. Sci. Technol. 5, 30 (1990).

17. T. Elliott, Proc. SPIE 3436, 763 (1998).

18. J. Piotrowski and A. Piotrowski, Opto-Electron. Rev. 14, 37 (2006).

19. Z. Djuric and J. Piotrowski, Opt. Eng. 31, 1955 (1992).

20. J. Piotrowski, W. Gawron, and Z. Djuric, Opt. Eng. 33, 1413 (1994).

21. S. Maimon and G. Wicks, Appl. Phys. Lett. 89, 151109 (2006).

22. P. Klipstein, Proc. SPIE 6940, 69402U (2008).

23. P. Klipstein, O. Klin, S. Grossmann, N. Snapi, B. Yaakobovitz, M. Brumer, I. Lukomsky, D. Aronov, M. Yassen, B. Yofis, A. Glozman, T. Fishman, E. Berkowitz, O. Maen, I. Shtrichman, and E. Weiss, Proc. SPIE 8012, 80122R (2011).

24. D.Z. Ting, A. Soibel, J. Nguyen, C.J. Hill, S.A. Keo, J.M. Mumolo, and S.D. Gunapala, Appl. Phys. Lett. 95, 023508 (2009).

25. M. Kopytko, K. Jóźwikowski, P. Madejczyk, W. Pusz, and A. Rogalski, Infrared Phys. Technol. 61, 162 (2013).

26. M. Kopytko, A. Kębłowski, W. Gawron, and W. Pusz, Semicond. Sci. Technol. 31, 035025 (2016). 
27. A. Piotrowski, P. Madejczyk, W. Gawron, K. Kłos, M. Romanis, M. Grudzień, J. Piotrowski, and A. Rogalski, OptoElectron. Rev. 12, 453 (2004).

28. W. Van Roosbroeck, Bell Syst. Tech. J. 29, 560 (1950).

29. M. Kurata, Numerical Analysis of Semiconductor Devices (Lexington: Lexington Books, D.C. Heath and Company, 1982).

30. T.J. Phillips and N.T. Gordon, J. Electron. Mater. 25, 1151 (1996).

31. M. Kopytko, K. Jóźwikowski, A. Rogalski, and A. Jóźwikowska, Opto-Electron. Rev. 18, 277 (2010).

32. J. Pawluczyk, J. Piotrowski, W. Pusz, A. Koźniewski, Z. Orman, W. Gawron, and A. Piotrowski, J. Electron. Mater. 44, 3163 (2015).
33. J.F. Siliquini, K.A. Fynn, B.D. Nener, L. Faraone, and R. Hartley, Semicond. Sci. Tech. 9, 1515 (1994).

34. J.F. Siliquini and L. Faraone, Infrared Phys. Technol. 38, 205 (1997).

35. P. Davis, C.T. Elliott, and A.M. White, Infrared Phys. 31, 575 (1991).

36. J. Piotrowski, Unpublished results (2008).

37. J. Piotrowski, K. Kłos, W. Gawron, J. Pawluczyk, Z. Orman and J. Piotrowski, in Proceedings of the SPIE 6542, 65421B1 (2007).

38. J. Piotrowski, W. Gawron, Z. Orman, J. Pawluczyk, K. Kłos, D. Stępień, and A. Piotrowski, Proc. SPIE 7660, 766031 (2010). 